\title{
Do Times until Treatment for Foreign Body Aspiration Relate to Complications?
}

\author{
Walailak Tatsanakanjanakorn and Surapol Suetrong \\ Department of Otorhinolaryngology, Faculty of Medicine, Khon Kaen University, Khon Kaen 40002, Thailand \\ Correspondence should be addressed to Surapol Suetrong; sursue@yahoo.com
}

Received 4 May 2016; Revised 29 June 2016; Accepted 27 July 2016

Academic Editor: David W. Eisele

Copyright (C) 2016 W. Tatsanakanjanakorn and S. Suetrong. This is an open access article distributed under the Creative Commons Attribution License, which permits unrestricted use, distribution, and reproduction in any medium, provided the original work is properly cited.

\begin{abstract}
Introduction. Foreign body aspiration is an emergency condition and may be fatal. Delayed diagnosis and treatment may be associated with complications. This study evaluated the association between time until treatment and complications due to foreign body aspiration. Methods. This study was a retrospective study conducted at Khon Kaen University Hospital, Thailand. We enrolled patients diagnosed with foreign body aspiration with evidence of foreign body detected using direct laryngobronchoscopy at any area from the larynx to the bronchus. Descriptive statistics were used to analyze the association of times of treatment with complications of foreign body aspiration. Results. During the study period, there were 43 patients that met the study criteria. The most common age group was $0-2$ years. Plant seeds were the most common foreign bodies (41.9\%), and the right main bronchus was the most common site (16 patients, $37.2 \%)$. There were 30 patients $(69.8 \%)$ that experienced complications from foreign body aspiration. Pneumonia was the most common complication (14 patients, 32.6\%). The retention time was not significantly associated with the presence of complications ( $p$ value: 0.366 ). Two patients $(4.7 \%)$ died due to complete airway obstruction and prolonged hypoxia. Conclusion. Times until treatment were not significantly associated with complications from foreign body aspiration.
\end{abstract}

\section{Introduction}

Foreign body aspiration (FBA) is an emergency condition and may be fatal [1]. This condition is more common in children. It has been estimated that 500 children die from foreign body aspiration each year in the USA [2]. The overall mortality rate of foreign body aspiration is approximately 5$7 \%$ [3]. FBA with complete airway obstruction is responsible for majority of the mortality cases immediately after the injuries [1, 4-7]. Although FBA with incomplete airway obstruction is not a cause of instant death, it can result in significant morbidities, complications, and delayed deaths [8]. The complications may be associated with many risk factors: foreign body (FB) sites, FB characteristics (size, shape, surface, edge, consistency, and types: organic, inorganic), child's characteristics, socioeconomic status, delayed diagnosis, and delayed management $[9,10]$. Delayed diagnosis and management are the main causes of serious complications regarding foreign body aspiration, particularly in children [11, 12]. The main reason for misdiagnosis or delayed diagnosis is a lack of specific signs or symptoms. About $15-20 \%$ of patients may not have any signs or symptoms of foreign body aspiration [13], while others may have symptoms that mimic those of pneumonia or asthma [14-18].

The complication rate of foreign body aspiration in children varies among studies from 14.6 to $27.8 \%[19,20]$. Pneumonia and respiratory distress were common complications. There are limited data on risk factors associated with complications of foreign body aspiration in children. A report from China [20] showed that the type of foreign body and symptoms were associated with respiratory complications. This study aimed to evaluate whether or not delayed treatment may be a risk factor for complications of foreign body aspiration.

\section{Method}

This study was a retrospective study conducted at Khon Kaen University Hospital, Thailand. The study period was between January 1997 and December 2006. We enrolled patients diagnosed with foreign body aspiration with evidence of foreign bodies detected using direct laryngobronchoscopy 
at any area from the larynx to the bronchus. Patients were excluded if medical records were incomplete or if the foreign body was found elsewhere such as the nose, nasopharynx, or esophagus.

Medical records of all eligible patients were reviewed. Demographic data, presenting symptoms, clinical findings, types of foreign bodies, and complications stemming from foreign body aspiration were recorded. Descriptive statistics were used to analyze clinical features and reported as percentage. The association between times until treatment and presence of complications was tested by Fisher's exact test using SPSS Statistics 17.0 software. $p$ values less than 0.05 were considered as statistically significant.

\section{Results}

During the study period, there were 103 patients diagnosed with foreign body aspiration. Among the 60 patients who were excluded, 28 were excluded due to nonlaryngotracheopulmonary FB, 17 were excluded due to incomplete medical records, and 15 were excluded due to the fact that no FB was identified. In total, 43 patients met the study criteria.

Clinical features are listed in Table 1. The most common age group was $0-2$ years. Coughing $(72.1 \%)$ and choking (67.4\%) were the most two common presenting symptoms, while decreased breath sound was the most common physical sign (62.8\%). Hyperaeration was the most common CXR finding (39.5\%). Normal CXR was found in 8 patients $(18.6 \%)$.

According to bronchoscopic findings (Table 2), plant seeds were the most common foreign bodies (41.9\%), and the right main bronchus was the most common site (16 patients, $37.2 \%)$.

There were 30 patients (69.8\%) who experienced complications from foreign body aspiration (Table 3). Pneumonia was the most common complication (14 patients, $32.6 \%$ ).

The retention times ranged from less than 24 hours to more than 15 days. Over a half of patients $(62.5 \%)$ had a retention time of more than 24 hours (Table 4 ).

The patients with retention time of more than 24 hours had over 2 times more complications than those with retention time of less than 24 hours (15:6; Table 4). But there was no significant association between times until treatment and presence of complications ( $p$ value of 0.366 by Fisher's exact test).

Two patients (4.7\%) died from complete airway obstruction and prolonged hypoxia. One patient aspirated chicken bone and had immediate respiratory failure for 20 minutes with cardiac arrest at the community hospital, at which bronchoscopy was not available. The other patient suffered from foreign body aspiration at the right main bronchus with failure to remove the foreign body by bronchoscopy at the local general hospital. During the transfer, the patient developed sudden severe airway obstruction for several hours prior to cardiac arrest.

\section{Discussion}

This was a retrospective study conducted at the referral center in northeast Thailand using data collected over a 10-year
TABLE 1: Clinical characteristics of patients with foreign body aspiration $(n=43)$.

\begin{tabular}{|c|c|c|}
\hline Characteristics & Frequency & Percentage \\
\hline \multicolumn{3}{|l|}{ Age, years } \\
\hline $0-2$ & 25 & 58.1 \\
\hline $3-7$ & 5 & 11.6 \\
\hline $8-12$ & 6 & 14.0 \\
\hline$>12$ & 7 & 16.3 \\
\hline \multicolumn{3}{|l|}{ Presenting symptoms* } \\
\hline Cough & 31 & 72.1 \\
\hline Choking & 29 & 67.4 \\
\hline Fever & 7 & 16.3 \\
\hline Hoarseness & 3 & 7.0 \\
\hline Others, that is, vomiting and sore throat & 3 & 7.0 \\
\hline \multicolumn{3}{|l|}{ Physical signs* } \\
\hline Decreased breath sound & 27 & 62.8 \\
\hline Rhonchi & 9 & 20.9 \\
\hline Wheezing & 9 & 20.9 \\
\hline Stridor & 4 & 9.3 \\
\hline Cyanosis & 1 & 2.3 \\
\hline Chest retraction & 5 & 11.6 \\
\hline Normal physical exam & 7 & 16.3 \\
\hline \multicolumn{3}{|l|}{ Chest X-ray } \\
\hline Hyperaeration & 17 & 39.5 \\
\hline Atelectasis & 7 & 16.3 \\
\hline Pulmonary infiltration & 2 & 4.7 \\
\hline Radioopacity & 4 & 9.3 \\
\hline Normal & 8 & 18.6 \\
\hline No data & 5 & 11.6 \\
\hline
\end{tabular}

Note: $*$ indicates that one patient may have more than one symptom/sign.

TABLE 2: Types and sites of foreign body aspiration in children $(n=$ 43).

\begin{tabular}{lcc}
\hline & Frequency & Percentage \\
\hline Types of foreign body & 18 & \\
Plant seeds & 9 & 41.9 \\
Peanut seed & 3 & 20.9 \\
Fish bone & 2 & 7.0 \\
Chicken bone & 3 & 4.7 \\
Tooth & 2 & 7.0 \\
Pork belly & 1 & 4.7 \\
Coin & 5 & 2.3 \\
Others* & & 11.6 \\
\hline Sites of foreign body & 16 & \\
Right main bronchus & 12 & 37.2 \\
Left main bronchus & 3 & 27.9 \\
Right second bronchus & 3 & 7.0 \\
Left second bronchus & 6 & 7.0 \\
Glottis & 1 & 14.0 \\
Trachea & 2 & 2.3 \\
Multiple sites & 4.7 \\
\hline
\end{tabular}

Note: $*$ included pen caps, a candy, a whistle, marbles, and a T-tube. 
TABLE 3: Complications of foreign body aspiration in children $(n=43)$.

\begin{tabular}{lcc}
\hline Complications & Frequency & Percentage \\
\hline Pneumonia & 14 & 32.6 \\
Hypoxia & 9 & 20.9 \\
Ventilatory support & 6 & 14.0 \\
Laryngeal stenosis & 1 & 2.3 \\
\hline
\end{tabular}

TABLE 4: Times until treatment and presence of complications $(n=40$; no data $=3)$.

\begin{tabular}{lccc}
\hline Times until treatment & With complications & Without complications & Total \\
\hline$<24$ hours & 6 & 9 & 15 \\
$1-2$ days & 5 & 3 & 8 \\
$3-7$ days & 3 & 5 & 8 \\
$8-14$ days & 2 & 0 & 2 \\
$>15$ days & 5 & 2 & 7 \\
\hline Total & 21 & 19 & 40 \\
\hline
\end{tabular}

period. Clinical features of patients with foreign body aspiration were comparable to those described in previous studies $[11,12]$. Most patients were young children with nonspecific symptoms/signs/and CXR findings [21]. A history of choking, poor air entry on examination, localized wheezing, and localized atelectasis may suggest foreign body aspiration. Note that normal CXR was found in $18.6 \%$ of patients (Table 1 ). CT may be helpful in the diagnosis of foreign body aspiration in cases of normal CXR [20].

The mortality rate in this study was $4.7 \%$, which was higher than those in previous reports $[22,23]$. Both of these previous reports were from India. The first one looked at 37 children who suffered from foreign body aspiration with no mortality, while the second report involved 140 children with a mortality rate of $0.7 \%$. Our mortality rate was high, because we included the fatality cases of primary and secondary hospital setting. These 2 patients died before reaching our facilities, the death of whom might be explained by lacking of proper medical instruments, inadequate experiences of physicians managing this problem, or severity (complete or nearly complete obstruction) of disease itself. However, our mortality rate was still in the global range (5-7\%) reported by Foltran et al. [3]. Bamber et al. [1] reported that $60 \%$ of the mortality cases died immediately after the event with no time for resuscitation, as did one of the cases we examined. Complete or near-complete airway obstruction was the primary mechanism by which this occurred. The remaining (40\%) had a prolonged survival time after treatment before death which is caused by hypoxic ischemic injury [1] just as another case of our study. Other mechanisms [24] included reflex cardiopulmonary arrest triggered by vagal stimulation and the esophageal foreign body compressing the posterior membranous trachea, causing airway obstruction. Delayed treatment due to a lack of recognition may be another factor associated with death or complications [3]. Our data (Table 4) showed no significant association between times until treatment and numbers of patients with complications from foreign body aspiration. However, complication rate was over 2 times higher in prolonged retention time of more than 24 hours. This was comparable to the study of Shlizerman et al. [11] in which rate of complications increased twofold for patients with delayed treatment time more than 2 days. But this increased complication was not statistically significant.

There are some limitations in this study. First, the sample size was quite small, despite the fact that this hospitalbased study was performed at the university tertiary care hospital. This may explain the low prevalence of foreign body aspiration. Also, some data were missing due to the data collection being retrospective. Further prospective studies are required to evaluate the association between times of treatment and complications of foreign body aspiration. This study evaluated the association of times until treatment and overall complications and was not specific to individual complications, as shown in Table 3.

\section{Conclusion}

Time until treatment was not significantly associated with complications from foreign body aspiration.

\section{Competing Interests}

The authors declare that there are no competing interests regarding the publication of this paper.

\section{Acknowledgments}

The authors would like to thank Associate Professor Kittisak Sawanyawisuth, Department of Medicine, Faculty of Medicine, Khon Kaen University. They would also like to thank manuscript writing clinic and Mr. Dylan Southard, English editor, for kind assistance in preparation of the paper. 


\section{References}

[1] A. R. Bamber, J. Pryce, M. Ashworth, and N. J. Sebire, "Fatal aspiration of foreign bodies in infants and children," Fetal and Pediatric Pathology, vol. 33, no. 1, pp. 42-48, 2014.

[2] R. Grassi, A. Faggian, F. Somma, C. N. De Cecco, A. Laghi, and F. Caseiro-Alves, "Application of imaging guidelines in patients with foreign body ingestion or inhalation: literature review," Seminars in Ultrasound, CT and MRI, vol. 36, no. 1, pp. 48-56, 2015.

[3] F. Foltran, S. Ballali, H. Rodriguez et al., "Inhaled foreign bodies in children: a global perspective on their epidemiological, clinical, and preventive aspects," Pediatric Pulmonology, vol. 48, no. 4, pp. 344-351, 2013.

[4] R. E. Mittleman, "Fatal choking in infants and children," The American Journal of Forensic Medicine and Pathology, vol. 5, no. 3, pp. 201-210, 1984.

[5] C. A. Ryan, W. Yacoub, T. Paton, and D. Avard, "Childhood deaths from toy balloons," American Journal of Diseases of Children, vol. 144, no. 11, pp. 1221-1224, 1990.

[6] A. Skevas, K. Karenzos, G. Exarchakos, and J. Kastanioudakis, "Cerebral anoxia with fatal outcome caused by aspiration of an air-filled balloon," HNO, vol. 39, no. 2, pp. 73-74, 1991 (German).

[7] B. D. Lifschultz and E. R. Donoghue, "Deaths due to foreign body aspiration in children: the continuing hazard of toy balloons," Journal of Forensic Sciences, vol. 41, no. 2, pp. 247-251, 1996.

[8] Y. Li, W. Wu, X. Yang, and J. Li, “Treatment of 38 cases of foreign body aspiration in children causing life-threatening complications," International Journal of Pediatric Otorhinolaryngology, vol. 73, no. 12, pp. 1624-1629, 2009.

[9] D. Passali, D. Gregori, G. Lorenzoni et al., "Foreign body injuries in children: a review," The American Journal of Forensic Medicine and Pathology, vol. 35, no. 4, pp. 265-271, 2015.

[10] A. M. Salih, M. Alfaki, and D. M. Alam-Elhuda, "Airway foreign bodies: a critical reviewfor a common pediatric emergency," World Journal of Emergency Medicine, vol. 7, no. 1, pp. 5-12, 2016.

[11] L. Shlizerman, S. Mazzawi, Y. Rakover, and D. Ashkenazi, "Foreign body aspiration in children: the effects of delayed diagnosis," American Journal of Otolaryngology, vol. 31, no. 5, pp. 320324, 2010.

[12] F. Karakoç, B. Karadağ, C. Akbenlioğlu et al., "Foreign body aspiration: what is the outcome?" Pediatric Pulmonology, vol. 34, no. 1, pp. 30-36, 2002.

[13] B. Gerbaka, J. Azar, and B. Rassi, "Foreign bodies of the respiratory tract in children. A retrospective study of 100 cases," Le Journal Médical Libanais, vol. 45, no. 1, pp. 10-18, 1997.

[14] M. François, Thach-Toan, D. Maisani, C. Prévost, and P. Roulleau, "Endoscopy for exploration for foreign bodies of the lower respiratory tract of the child. Apropos of 668 cases," Annales d'Otolaryngologie et de Chirurgie Cervico-Faciale, vol. 102, no. 6, pp. 433-441, 1985.

[15] L. Even, N. Heno, Y. Talmon, E. Samet, Z. Zonis, and A. Kugelman, "Diagnostic evaluation of foreign body aspiration in children: a prospective study," Journal of Pediatric Surgery, vol. 40, no. 7, pp. 1122-1127, 2005, Erratum in: Journal of Pediatric Surgery, vol. 40, no. 11, pp. 1815, 2005.

[16] C.-Y. Chiu, K.-S. Wong, S.-H. Lai, S.-H. Hsia, and C.-T. Wu, "Factors predicting early diagnosis of foreign body aspiration in children," Pediatric Emergency Care, vol. 21, no. 3, pp. 161-164, 2005.
[17] A. B. Chang, "Pediatric cough: children are not miniature adults," Lung, vol. 188, supplement 1, pp. S33-S40, 2010.

[18] B. S. Sharma, D. S. Shekhawat, P. Sharma, C. Meena, and H. Mohan, "Acute respiratory distress in children: croup and acute asthma," Indian Journal of Pediatrics, vol. 82, no. 7, pp. 629-636, 2015.

[19] A. Jaswal, U. Jana, and P. K. Maiti, "Tracheo-bronchial foreign bodies: a retrospective study and review of literature," Indian Journal of Otolaryngology and Head \& Neck Surgery, vol. 66, supplement 1, pp. 156-160, 2014.

[20] Y. H. Yang, X. G. Zhang, J. L. Zhang, Y. B. Zhang, and C. P. Kou, "Risk factors for preoperative respiratory complications in children with tracheobronchial foreign bodies," The Journal of International Medical Research, vol. 44, no. 2, pp. 338-345, 2016.

[21] A. I. Naragund, R. S. Mudhol, A. S. Harugop, P. H. Patil, P. S. Hajare, and V. V. Metgudmath, "Tracheo-bronchial foreign body aspiration in children: a one year descriptive study," Indian Journal of Otolaryngology and Head \& Neck Surgery, vol. 66, supplement 1, pp. 180-185, 2014.

[22] p. parida, N. S. Nirmal, S. Gopalakrishnan, and S. Saxena, "Factors predicting early diagnosis of pediatric laryngotracheobronchial foreign bodies," Otolaryngologia Polska, vol. 69, no. 6, pp. 31-35, 2015.

[23] A. Williams, C. George, S. Sam, S. Shukla, and P. Atul, "An audit of morbidity and mortality associated with foreign body aspiration in children from a tertiary level hospital in Northern India," African Journal of Paediatric Surgery, vol. 11, no. 4, p. 287, 2014.

[24] R. W. Byard, "Mechanisms of unexpected death in infants and young children following foreign body ingestion," Journal of Forensic Sciences, vol. 41, no. 3, pp. 438-441, 1996. 


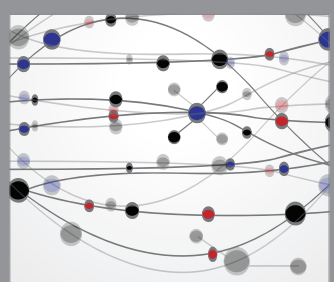

The Scientific World Journal
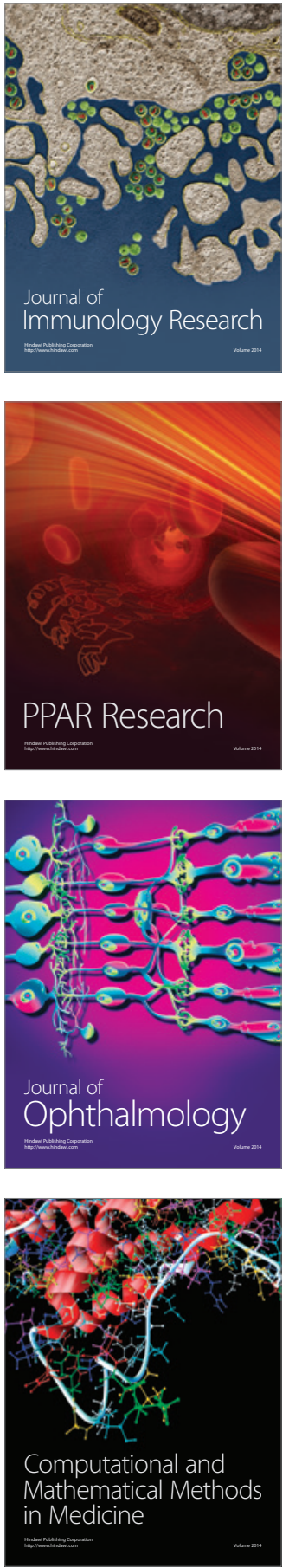

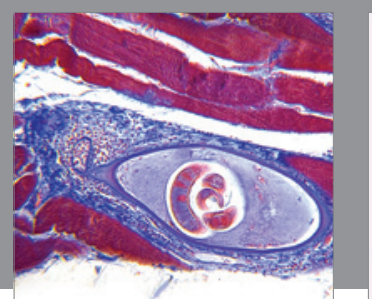

Gastroenterology Research and Practice

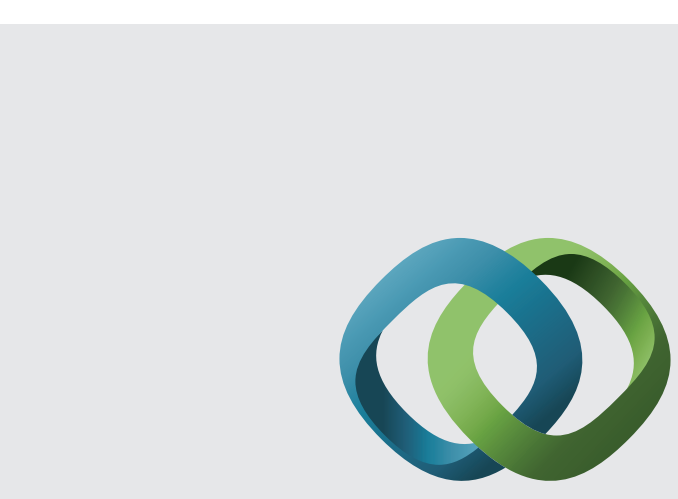

\section{Hindawi}

Submit your manuscripts at

http://www.hindawi.com
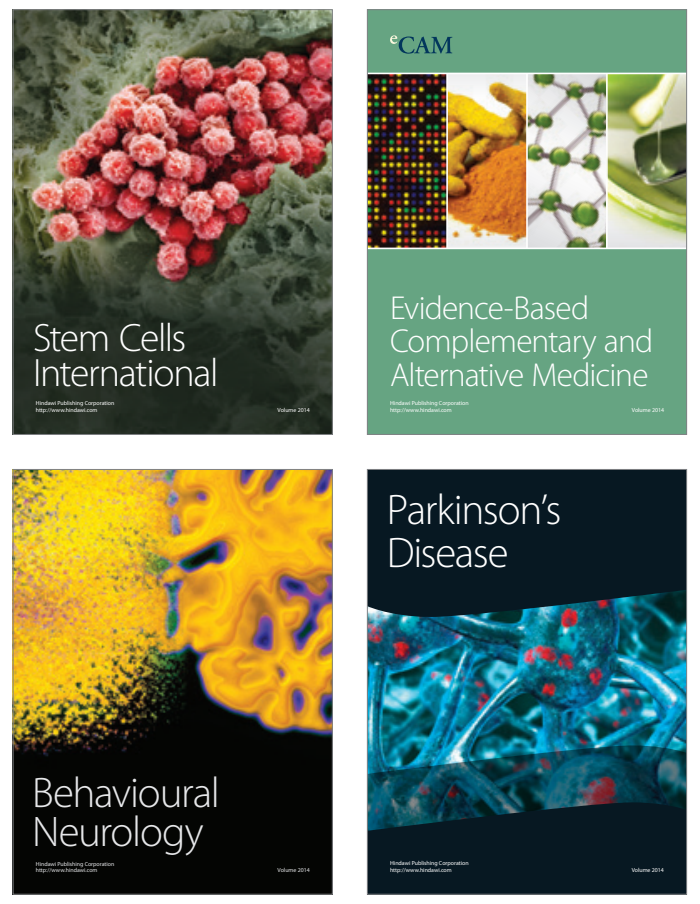
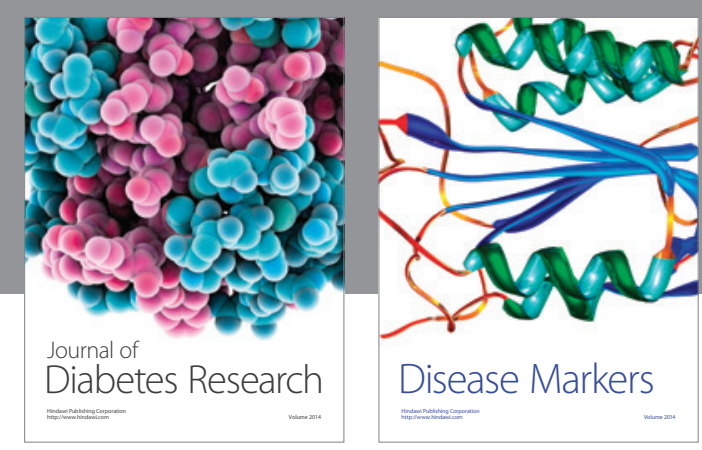

Disease Markers
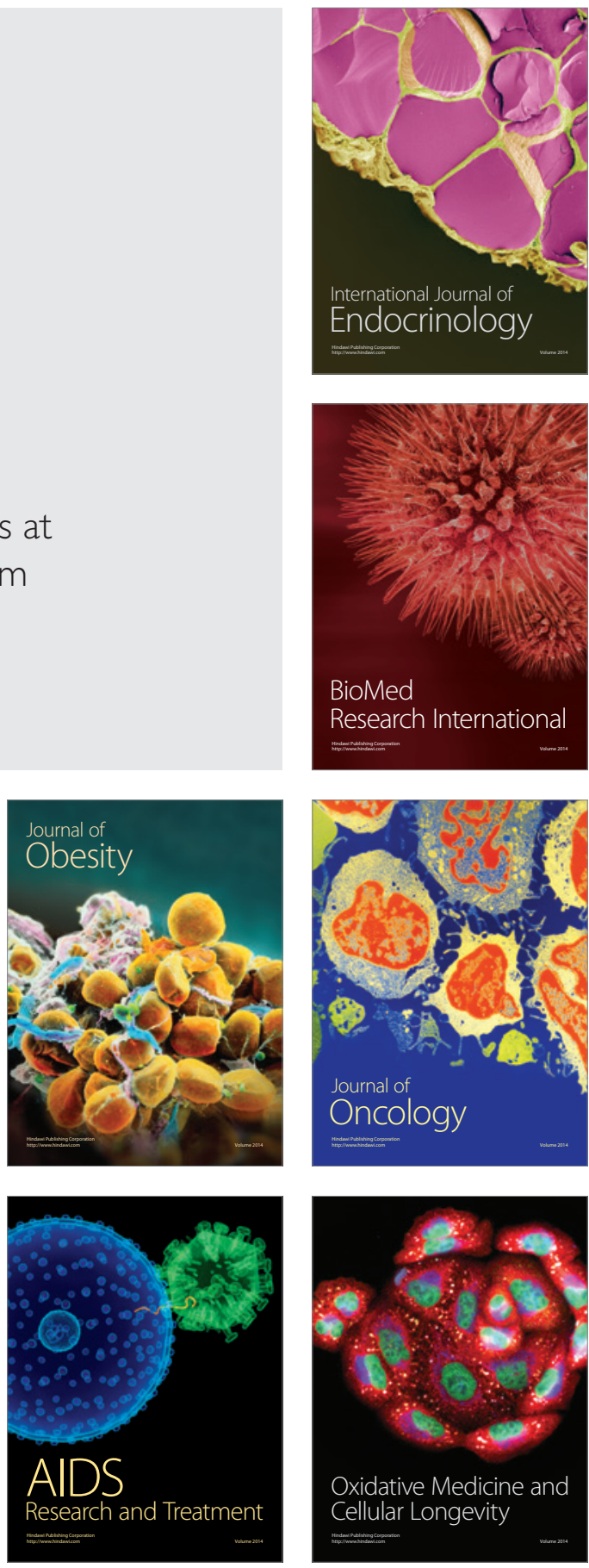